\title{
Circuit
}

Musiques contemporaines

\section{Catalogue des oeuvres commandées par la SMCQ}

\section{Solenn Hellégouarch}

Volume 27, numéro 2, 2017

Souvenirs du futur : pour les 50 ans de la SMCQ

URI : https://id.erudit.org/iderudit/1040877ar

DOI : https://doi.org/10.7202/1040877ar

Aller au sommaire du numéro

Éditeur(s)

Circuit, musiques contemporaines

ISSN

1183-1693 (imprimé)

1488-9692 (numérique)

Découvrir la revue

Citer ce document

Hellégouarch, S. (2017). Catalogue des oeuvres commandées par la SMCQ. Circuit, 27(2), 53-66. https://doi.org/10.7202/1040877ar d'utilisation que vous pouvez consulter en ligne.

https://apropos.erudit.org/fr/usagers/politique-dutilisation/ 


\section{Catalogue des œuvres commandées par la SMCQ}

Solenn Hellégouarch

Ce catalogue présente les œuvres commandées (ou co-commandées) par la Société de musique contemporaine du Québec (SMCQ) entre 1968 et 2017. Les œuvres sont classées par année de création, puis par date de création ${ }^{1}$. La présence d'un astérisque indique que la commande a reçu l'aide du Conseil des arts du Canada (CAC).

\section{8}

- *Gilles Tremblay, Souffles (Champs II) (1968), z flûtes (et piccolo), hautbois, clarinette, cor, 2 trompettes en si bémol (et trompettes en do), 2 trombones, piano, 2 percussions et contrebasse. Création : 21 mars, Concert 11, Salle Claude-Champagne (Montréal); Ensemble de la SMCQ, Serge Garant, direction.

\section{9}

- "Norma Beecroft, Rasas I (1968), flûte, harpe, piano, percussions, violon, alto et violoncelle. Création: 9 janvier, Concert 16, Salle Claude-Champagne (Montréal); Groupe instrumental de Montréal, Serge Garant, direction.

\section{0}

- "Jacques Hétu, Cycle, op. 16 (1969), flûte, clarinette, clarinette basse, basson, cor, 2 trompettes, 2 trombones et piano. Création: 5 février, Concert 22, Théâtre Maisonneuve (Montréal); Groupe instrumental de Montréal, Serge Garant, direction.

\section{1}

- *R. Murray Schafer, Music for the Morning of the World (1971), mezzo-soprano et bande. Création: 25 février, Concert 33, Théâtre Maisonneuve (Montréal); Phyllis Mailing, mezzo-soprano, Groupe instrumental de Montréal, Serge Garant, direction.
1. Dès lors, seules les œuvres créées ont été retenues dans le présent catalogue. 
- *John Hawkins, Waves (1971), soprano et piano. Création: 4 novembre, Concert 37 , Salle Claude-Champagne (Montréal); Margo MacKinnon, soprano, Bruce Mather, piano, Ensemble de la SMCQ, Serge Garant, direction.

\section{2}

- "François Morel, Iikkii (froidure) (1972), flûte (et piccolo et flûte en sol), hautbois, clarinette, basson, cor, trompette, trombone, tuba, guitare, harpe, piano (et célesta), 2 percussions, violon, alto, violoncelle et contrebasse. Création : 3 février, Concert 39, Salle Claude-Champagne (Montréal); Ensemble de la SMCQ, Serge Garant, direction.

- "Serge Garant, Circuits II (1973), 2 flûtes (piccolos et flûte en sol), clarinette, cor, trompette (et trompette piccolo), trombone, harpe, 2 pianos, 3 percussions, violon et alto. Création: 31 mars, Concert 43, Théâtre du Casino municipal (Royan, France); Ensemble de la SMCQ, Serge Garant, direction.

\section{3}

- "Robert Aitken, Lalitá: Shadows II (1973), flûte solo, 2 harpes, 3 violoncelles et 2 percussions. Création : 16 janvier, Concert 48, Conservatoire royal de Bruxelles; Ensemble de la SMCQ, Serge Garant, direction.

- *Bruce Mather, Madrigal V (1972-1973), soprano, contralto, flûte (et piccolo), hautbois, clarinette (et clarinette basse), cor, trompette, mandoline, harpe, orgue électrique, piano, 2 percussions, 2 violons, alto, violoncelle et contrebasse. Création: 12 avril, Concert 52, Salle Claude-Champagne (Montréal); Ann Golden, contralto, Margo MacKinnon, soprano, Ensemble de la SMCQ, Serge Garant, direction.

- "Jean Papineau-Couture, Obsession (1973), 3 flûtes, hautbois, clarinette, basson, cor, piano, 2 percussions, 2 violons, 2 alti et 2 violoncelles. Création: 18 octobre, Concert 54, Salle Claude-Champagne (Montréal); Ensemble de la SMCQ, Serge Garant, direction.

\section{4}

- "Micheline Coulombe Saint-Marcoux, Ishuma (1973), voix, trombone, synthétiseur, ondes Martenot, orgue Hammond, 3 percussions et violon. Création : 21 mars, Concert 58, Salle Claude-Champagne (Montréal); Marthe Forget, soprano, Ensemble de la SMCQ, Serge Garant, direction.

- "Gilbert Amy, Sonata pian'e forte (1974), soprano, mezzo-soprano, flûte, clarinette basse, cor, cor anglais, guitare, mandoline, harpe, 2 pianos, marimba, xylophone, jeu de cloches, crotales, alto et violoncelle. Création: 18 avril, Concert 59, Salle Claude-Champagne (Montréal); Ensemble de la SMCQ, Gilbert Amy, direction.

\section{5}

- *alcides lanza, plectros IV (1974), 2 pianos. Création: 23 janvier, Concert 65 (Robert Leroux), Salle Claude-Champagne (Montréal) ; Pierrette Lesage et Bruce Mather, pianos. 
- *Brian Cherney, Concerto de chambre (1974), alto solo, flûte, clarinette, cor, trompette, trombone, harpe, percussions, violon, violoncelle et contrebasse. Création : 27 mars, Concert 67, Salle Claude-Champagne (Montréal); Osher Green, alto, Ensemble de la SMCQ, Serge Garant, direction.

- "Claude Vivier, Liebesgedichte (1975), 4 voix mixtes, hautbois, clarinette, 2 bassons dont un remplaçant le heckelphone, cor anglais, 2 trompettes, trombone et trombone basse. Création : 2 octobre, Concert 7o, Salle Pollack (Montréal); Ensemble de la SMCQ, Serge Garant, direction.

\section{6}

- *Marcelle Deschênes, Moll, opéra lilliput pour six roches molles (version réduite, 1976), 2 clarinettes, 3 trombones, 3 groupes de percussions, jouets d'enfant amplifiés et bande stéréo. Création : 18 mars, Concert 75, Salle Pollack (Montréal); Ensemble de la SMCQ, Serge Garant, direction.

- *Iannis Xenakis, Épéi (puisque, depuis, since) (1976), clarinette en si bémol, cor anglais, trompette en $d o, 2$ trombones et contrebasse. Création: 9 décembre, Concert 88, Salle Pollack (Montréal); Ensemble de la SMCQ, Serge Garant, direction.

\section{7}

- "Otto Joachim, Uraufführung (1977), quatuor à cordes, 3 flûtes, flûte basse, clarinette basse, basson, cor, guitare et percussions. Création: 10 mars, Concert 9o, Salle Pollack (Montréal); David Joachim, guitare, Ensemble de la SMCQ, Serge Garant, direction.

- "Clermont Pépin, Interactions (1977), 2 pianos et 7 groupes de percussion. Création : 14 avril, Concert 92, Salle Pollack (Montréal); Ensemble de la SMCQ, Serge Garant, direction.

- *Donald Steven, Images (Refractions of Time and Space) (1977), flûte électrique, piano électrique, 2 percussions et contrebasse électrique. Création : 27 octobre, Concert 93, Salle Pollack (Montréal); Ensemble de la SMCQ, Serge Garant, direction.

\section{9}

- Michel Gonneville, Variations Auras (1978), flute, clarinette basse, cor anglais, cor, trompette, trombone, violon, alto et violoncelle. Création : 22 février, Concert 112, Salle Pollack (Montréal); Ensemble de la SMCQ, Serge Garant, direction².

- "Denis Lorrain, Di mi se mai... (1979), cor, trompette en ré, trompette en do, trombone, tuba, percussions et support. Création: 6 mars, Concert 119, Salle Pollack (Montréal); Ensemble de la SMCQ, Serge Garant, direction.

\section{0}

- *Bengt Hambraeus, Relief - haut et bas (1979), 2 flutes, 3 cors, trombone, 2 percussions et 2 contrebasses. Création : 10 avril, Concert 120, Salle Pollack (Montréal); Ensemble de la SMCQ, Serge Garant, direction.
2. L'œuvre fait l'objet d'une révision en 1986. 
- "John Burke, À la source d'Hypocrène (1981), 2 flûtes, clarinette, trompette, harpe, clavecin, célesta, 2 percussions, violon, alto et violoncelle. Création : 29 janvier, Concert 127, Salle Pollack (Montréal); Ensemble de la SMcQ, Serge Garant, direction.

- "Yves Daoust, Valse (1981), flûte/piccolo, clarinettes en si bémol et mi bémol, saxophones alto, ténor et baryton, trompette, orgue électrique, clavecin amplifié, piano, piano électronique, violon et bande stéréo. Création : 9 avril, Concert 130, Salle Pollack (Montréal); Ensemble de la SMCQ, Serge Garant, direction.

- Alain Lalonde, Espaces (1981), flûte (et piccolo), clarinettes en mi bémol, clarinette basse, clarinette contrebasse, basson (et contrebasson), 3 percussions, violon, contrebasse et système de spatialisation. Création: 10 décembre, Concert 133, Salle Pollack (Montréal); Ensemble de la SMCQ, Serge Garant, direction.

\section{2}

- Serge Provost, Anagramme (1981), piano. Commandée avec l'aide d'un don de Jean Éthier-Blais. Création : 28 janvier, Concert 134, Salle Pollack (Montréal); Louise Bessette, piano.

- Créations du 25 mars, Concert 136, Salle Pollack (Montréal):

- "Marius Constant, Précis de décomposition (1982), clarinette, basson, cor, 2 violons, alto, violoncelle, contrebasse et support. Ensemble de la SMCQ, Marius Constant, direction.

- "Michel Longtin, Kata: San Shi Ryu (1982), flûte, 5 percussions, 2 violoncelles et 2 contrebasses. Ensemble de la SMCQ, Serge Garant, direction.

- *André Prévost, Mutations (1982), 2 percussions, 4 violons, 2 altos, 2 violoncelles et 2 contrebasses. Ensemble de la SMCQ, Serge Garant, direction.

- *John Rea, Treppenmusik (1982), quatuor à cordes, quatuor de saxophones, clarinettes en si bémol et mi bémol, 2 clarinettes basses et délai. Création : 7 octobre, Concert 134, Salle Pollack (Montréal); Ensemble de la SMCQ, Serge Garant, direction.

- "Michel Longtin, Kata: San Shi Ryu (1982), flûte, 5 percussions, 2 violoncelles, 2 contrebasses. Création : 16 décembre, Concert 140, Salle Pollack (Montréal); Ensemble de la SMCQ, Serge Garant, direction.

\section{3}

- *Alexina Louie, Music for a Thousand Autumns (1983), 2 flutes (et piccolos), hautbois, clarinette, basson, piano, 2 percussions, violon, alto, violoncelle et contrebasse. Création : 6 octobre, Concert 145, Salle Pollack (Montréal); Ensemble de la SMCQ, Serge Garant, direction.

- "José Evangelista, Clos de Vie (1983), guitare électrique, banjo, harpe, clavecin, piano, percussions, 2 violons, violoncelle et contrebasse. Création: 3 novembre, Concert 146, Salle Pollack (Montréal); Ensemble de la SMcQ, Serge Garant, direction. 
- *Walter Boudreau, Incantations III (1984), 3 flûtes (et piccolos), hautbois (et cor anglais), clarinette en $m i$ bémol, clarinette en si bémol, clarinette basse et basson (et contrebasson). Création : 8 mars, Concert 150, Salle Pollack (Montréal); Ensemble de la SMCQ, Serge Garant, direction.

- *Pierre Trochu, Tangente (1984), flûte, hautbois (et cor anglais), clarinette, piano, 2 percussions, violon, alto, violoncelle et contrebasse. Création : 2 octobre, Concert 152, Salle Pollack (Montréal); Ensemble de la SMCQ, Serge Garant, direction.

- *Allan Bell, Gaia (1984), flûte, hautbois, clarinette, piano, 2 percussions, 4 violoncelles. Création : 15 novembre, Concert 153, Salle Pollack (Montréal); Ensemble de la SMCQ, Serge Garant, direction.

\section{5}

- "Makoto Shinohara, Tabiyuki (1984), mezzo-soprano, flûte (et piccolo, et flûte en sol, sifflet à coulisse), hautbois (et cor anglais), clarinette (et clarinette basse), basson, cor, trompette, trombone, percussions, violon, alto, violoncelle et contrebasse. Création : 28 février, Concert 156, Salle Pollack (Montréal); Jocelyne Coutu-Fleury, mezzo-soprano, Ensemble de la SMCQ, Serge Garant, direction.

- "Serge Arcuri, Prologue (1985), flûte, clarinette (et clarinette basse), saxophone soprano, cor, harpe, piano, percussions, contrebasse et support. Création: 10 octobre, Concert 159, Salle Pollack (Montréal); Ensemble de la SMcQ, Serge Garant, direction.

\section{6}

- "Denys Bouliane, ...À propos... et le Baron Perché? (1985), 2 clarinettes (et clarinettes basses), 2 bassons (et contrebassons), 2 cors, trompette, 2 trombones et contrebasse. Création : 13 mars, Concert 164, Salle Pollack (Montréal); Ensemble de la SMCQ, Serge Garant, direction.

- Richard Boucher, Durer (1986), soprano, flûte, hautbois, clarinette, z percussions, violon, violoncelle et contrebasse. Commandée avec l'aide de Maryvonne Kendergi. Création : 25 septembre, Concert 167, Salle Pollack (Montréal); Pauline Vaillancourt, soprano, Ensemble de la SMCQ, Walter Boudreau, direction.

- "José Evangelista, Merapi (1986), flûte, clarinette, harpe, vibraphone, violon, violoncelle et contrebasse. Création : 4 décembre, Concert 170, Salle Pollack (Montréal); Ensemble de la SMCQ, Marius Constant, direction.

\section{7}

- "Jean Papineau-Couture, Nuit polaire (1986), contralto, 2 flûtes (et piccolos, flûtes en sol, flûtes basses), hautbois (et cor anglais), harpe, piano, percussions, violon, alto, violoncelle et contrebasse. Création : 29 janvier, Concert 171, Salle Pollack (Montréal); Madeleine Jalbert, contralto, Ensemble de la SMCQ, Lorraine Vaillancourt, direction. 
- Créations du 16 avril, Concert 174, Salle Pollack (Montréal):

- *Raynald Arseneault (avec Kenneth Curie), The Aristocratic Lover (1985), baryton-ténor, 4 voix mixtes, hautbois (et cor anglais), 2 cors, piano, 4 violons, alto et violoncelle. Ensemble de la SMCQ, Gilles Auger, direction.

- *Ka Nin Chan, Nuage précieux (1987), hautbois (et cor anglais), clarinette, basson, cor, trompette, harpe, piano (et célesta), violon, alto et violoncelle. Ensemble de la SMCQ, Gilles Auger, direction.

- "Denis Gougeon, Heureux qui, comme... (1987), soprano, piccolo, cor anglais, saxophone baryton, percussion et quintette à cordes. Marie-Danielle Parent, soprano, Ensemble de la SMCQ, Gilles Auger, direction.

\section{8}

- *Yves Daoust, Variations sur un air d'accordéon! (1988), comédien-accordéoniste et bande stéréo. Création : 21 janvier, Concert 178, Studio-théâtre Alfred Laliberté (Montréal); Richard Dubé, mime.

- Créations du 14 février, Tournée canadienne, Jack Singer Concert Hall (Calgary):

- *Allan Gordon Bell, Concerto for Two Orchestras (1984), 3 flûtes, 3 hautbois, 3 clarinettes, 3 bassons, 4 cors, 3 trompettes, 2 pianos, 5 percussions, 12 violons, 4 altos, 4 violoncelles et 2 contrebasses. Commandée avec l'Esprit Orchestra. Esprit Orchestra et Ensemble de la SMCQ, Walter Boudreau et Alex Pauk, direction.

- "Walter Boudreau, Versus (1987), flûte, hautbois, clarinette, basson, 2 cors, trompette, trombone, piano et 3 percussions. Ensemble de la SMCQ, Walter Boudreau, direction.

- "John Burke, Far Calls. Coming, Far! (1987), flûte, hautbois, clarinette, basson, 2 cors, trompette, trombone, piano, percussions, vibraphone et marimba. Ensemble de la SMCQ, Walter Boudreau, direction.

- Alain Lalonde, Mouvances, errances et toute cette sorte d'ances (1988), 2 hautbois, 3 cors, 2 tubas, percussions électroniques et 4 altos. Commandée avec l'aide du ministère des Affaires culturelles du Québec. Création: 7 avril, Concert 185, Le Spectrum de Montréal; Ensemble de la SMCQ, Walter Boudreau, direction.

- *Rodney Sharman, The Proximity of Mars (1988), 2 flûtes, 2 cors, 2 trompettes, 2 trombones, harpe, piano, percussions, violon et violoncelle. Création : 11 novembre, Concert hors série, Salle Claude-Champagne (Montréal); Ensemble de la SMCQ, Walter Boudreau, direction.

\section{9}

- "John Oliver, Aller-Retour (1988), flûte, cor anglais, clarinette basse, cor, trompette, trombone, 2 percussions, violon, violoncelle et contrebasse. Création: 16 mars, Concert 19o, Salon ovale du Ritz-Carlton (Montréal); Ensemble de la SMCQ, Walter Boudreau, direction.

- "Francis Dhomont, Chroniques de la lumière (1989, arr. 1989), bande stéréo; sur une idée de l'artiste visuel Luc Courchesne (scénographie). Commandée avec 
l'aide du ministère des Affaires culturelles du Québec. Création : 26 avril, Doppler Concert (Concert 191), Le Spectrum de Montréal; Francis Dhomont, diffusion³.

- Créations du 9 novembre, Concert 192, Salle Claude-Champagne (Montréal):

- *Tim Brady, Shadow Painting (1989), flûte (et flûte alto), clarinette (et clarinette basse), 2 cors, harpe, percussions, alto et violoncelle. Ensemble de la SMCQ, Walter Boudreau, direction.

- Alain Thibault, Babel (concerto de chambre) (1989), flûte, clarinette basse, 2 trompettes, 2 trombones, 2 percussions, alto et violoncelle. Commandée avec l'aide du ministère des Affaires culturelles du Québec.

\section{0}

- Créations du 10 novembre, Concert 198, Salle Pollack (Montréal):

- *Steven Gellman, Red Shoes (1990), clarinette, basson, 2 cors, 2 trompettes, trombone, synthétiseur Yamaha DX-7, percussions, violon, alto et violoncelle. Ensemble de la SMCQ, Walter Boudreau, direction.

- *Michel Longtin, Citortia (1990), 2 flûtes (piccolos), hautbois, 2 clarinettes, clarinette basse, 2 bassons, 2 cors, 2 trompettes, 2 trombones, 2 synthétiseurs Yamaha DX-7, 2 percussions, 2 violons, alto, violoncelle et contrebasse. Ensemble de la SMCQ, Walter Boudreau, direction.

\section{1}

- *John Rea, Las Meninas (1990-1991), piano. Commandée à la demande du pianiste Yvar Mikhashoff. Création : 14 avril, Concert 202 [Yvar Mikhashoff], Théâtre PaulDesmarais (Montréal) ${ }^{4}$.

- "Gilles Tremblay, Musique du feu (1991), piano solo, flûte (et piccolo), 2 flûtes, 3 hautbois, clarinette (et clarinettes basses), clarinette (et clarinette en mi bémol), clarinette, 3 bassons, 4 cors, 3 trompettes, 2 trombones, trombone basse, 2 tubas et 6 percussions. Commandée à l'occasion du Concert Gala du $25^{\mathrm{e}}$ anniversaire de fondation de la SMCQ. Création : 2 juin, Concert 203, Hangar 9, Quai King-Edward (Montréal); Louise-Andrée Baril, piano, Ensemble de la SMCQ, Walter Boudreau, direction.

- Créations du 7 novembre, La mascarade et la fête, Salle Redpath (Montréal):

- Anonymes, Critique (1991), bande stéréo.

- Ned Bouhalassa, Le désir fauve (1991), bande stéréo.

- André Hamel, Théâtre navrant (1991), clarinette solo, guitare solo, soprano, saxophone ténor, trompette, trombone, clavier, percussions, violoncelle et contrebasse. Commandée avec l'aide du ministère des Affaires culturelles du Québec. Natalie Choquette, soprano, Robert Marcel Lepage, clarinette, René Lussier, guitare électrique, Ensemble de la SMCQ, Véronique Lacroix, direction.

- *James Harley, Étude sur une fête (1991), saxophone alto, trompette, trombone, clavier, violoncelle et contrebasse. Ensemble de la SMCQ, Véronique Lacroix, direction.
3. Le $3^{\mathrm{e}}$ mouvement, "Météores", a été modifié en 2005. Les deux autres mouvements sont respectivement intitulés "Miroitements" et "Artifices".

4. Un arrangement de Marie-Hélène Brault et Pamela Reimer est réalisé en 2015 pour piano et flûte. 
- "Marc Hyland, Quand le journal fermente comme un éclair claquemuré (1991), bande. Commandée avec l'aide du ministère des Affaires culturelles du Québec.

- *Daniel Leduc, Un bal masqué, bande stéréo.

- Estelle Lemire, Rimes et mesures dépourvues (1991), clarinette solo, guitare électrique solo, saxophone soprano, trompette, trombone, percussions, violoncelle, contrebasse et support. Commandée avec l'aide du ministère des Affaires culturelles du Québec. Robert Marcel Lepage, clarinette, René Lussier, guitare électronique, Ensemble de la SMCQ, Véronique Lacroix, direction.

- Silvio Palmieri, Erratum musical (music for non-thinkers) (1991), soprano colorature, clarinette, saxophone soprano, trompette, trombone, piano, percussions, violoncelle et contrebasse. Natalie Choquette, soprano, Ensemble de la SMCQ, Véronique Lacroix, direction.

- Marie Pelletier, C.S.Q.M. (1991), clarinette solo, guitare électrique solo, soprano, saxophone alto, trompette, trombone, clavier, percussions, violoncelle et contrebasse. Commandée avec l'aide du ministère des Affaires culturelles du Québec. Natalie Choquette, soprano, Robert Marcel Lepage, clarinette, René Lussier, guitare électronique, Ensemble de la SMcQ, Véronique Lacroix, direction.

\section{2}

- "Stephen Chatman, Creatures of Earth and Sky (1991), harpe. Création: 19 janvier, Concert 207, Théâtre Paul-Desmarais (Montréal); Nathalie Teevin-Lebens, harpe.

- Créations du 25 mars, Concert zo8, Église Saint-Jean-Baptiste (Montréal):

- "Jean Lesage, Babylone - La porte de Dieu (1991), orgue. Gisèle Guibord, orgue.

- *François Morel, Lumières sculptées (1992), 2 cors, 2 trompettes, 2 trombones, tuba et 3 percussions. Ensemble de la SMCQ, Walter Boudreau, direction.

- René Lussier, Destination Soleil (1992), guitare électrique solo, clarinette (et clarinette basse), basson, trompette, trombone basse, accordéon, 2 percussions, violon et contrebasse. Création : 22 octobre, Québec-Amérique (concert 210), Salle Pierre-Mercure (Montréal); René Lussier, guitare électrique, Ensemble de la SMCQ, Walter Boudreau, direction.

- Serge Provost, Églogue - Le jardin des oliviers (1992), flûte, trompette, harmonium, célesta, 3 percussions, 2 violons, alto et violoncelle. Création: 10 décembre, Québec-France (concert 212), Salle Pierre-Mercure (Montréal); Ensemble de la SMCQ, Walter Boudreau, direction.

\section{3}

- *Denys Bouliane, Paysage Qu. (1993), clarinette (et clarinette basse), 3 trompettes, trombone, trombone basse, 4 percussions, violon, alto, violoncelle et contrebasse. Création: 21 octobre, Québec-Pays-Bas (concert 216), Salle Pierre-Mercure (Montréal); Ensemble de la SMCQ, Walter Boudreau, direction. 
- *Michel-Georges Brégent, Dilmun-Eden: quatre règnes dans l'évolution terrestre (1981-1986), flûte (piccolo, flûte en sol), hautbois (cor anglais), 4 percussions, 2 synthétiseurs, 2 harpes, 2 violons, 2 altos et contrebasse. Création : 26 mai, QuébecUtopie (concert 221), Salle Pierre-Mercure (Montréal); Ensemble de la SMCQ, Walter Boudreau, direction.

\section{5}

- "Gilles Tremblay, L'arbre de Borobudur (1995), gamelan, ondes, 2 cors, 2 harpes, 2 percussions et contrebasse. Création : 9 mars, Créations + gamelan (concert 225), Salle Pierre-Mercure (Montréal); Evergreen Club Contemporary Gamelan5, Ensemble de la SMCQ, Walter Boudreau, direction.

- Créations du 11 mai, Journées XX siècle (concert 226), Salle Pierre-Mercure (Montréal) :

- *Walter Boudreau, Neuf Zéniths II (1994-1995), flûte (et piccolo), hautbois, clarinette (et clarinette basse), cor, trompette, trombone, piano, 2 percussions, violon, alto et violoncelle. Ensemble de la SMCQ, Walter Boudreau, direction.

- *Otto Joachim, Stacheldraht (1994), flûte solo, quatuor à cordes, récitante, hautbois, clarinette, basson, 3 cors, timbales et contrebasse. Denis Bluteau, flûte, Colombe Demers, récitante, Ensemble de la SmcQ, Walter Boudreau, direction.

- Créations du 8 juin, Électronie (concert 227), Salle Pierre-Mercure (Montréal):

- "Paul Dolden, The Heart Tears Itself Apart with the Power of its own Muscle: Resonance \#3 (1994), 4 violons, 2 altos, 2 violoncelles, 2 contrebasses et bande stéréo. Ensemble de la SMCQ, Walter Boudreau, direction ${ }^{6}$.

- *Alain Thibault, Le cauchemar climatisé (1995), marimba et support. Daniel Pancaldi, marimba.

\section{6}

- Yves Daoust, Impromptu (1994; arr. 1995), piano, synthétiseur (échantillonneur) et bande stéréo. Commandée pour l'Ensemble Grame, avec l'aide du Conseil des arts et lettres du Québec (CALQ). Création : 30 janvier, Live from Lyon (concert 229), Salle Pierre-Mercure (Montréal); Anne Gaudemer, clavier, Manuel Schweizer, piano ${ }^{7}$.

- *Marc Hyland, Afterdreamlingly (A Legend?) (1995-1996), soprano, flûte, clarinette, cor, piano, percussions, violon et violoncelle. Création : 15 février, Tribunes (concert 230), Salle Pierre-Mercure (Montréal); Yolande Parent, soprano, Ensemble de la SMCQ, Walter Boudreau, direction.

- "Linda Bouchard, Compressions (1996), 2 cors, 3 trompettes (et trompette piccolo), 3 trombones, percussions, harpe, piano, violon, alto, violoncelle et contrebasse. Création : 13 juin, Musica Maxima (concert 232), Salle Pierre-Mercure (Montréal); Ensemble de la SMCQ, Walter Boudreau, direction.

- *Ana Sokolović, Jeu des portraits (1996), flûte (et flûte en sol), hautbois (et cor anglais), clarinette, basson, cor, trompette, trombone, piano et 2 percussions.
5. Voir: http://evergreenclubgamelan. ca (consulté le 6 avril 2017).

6. Une version extensive est réalisée en 1997 et créée le 5 octobre de la même année par l'Esprit Orchestra, à Toronto, sous la direction d'Alex Pauk.

7. Un autre arrangement a été réalisé en 2014 par le compositeur. 
Création: 14 novembre, Anniversaires (concert 235), Salle Pierre-Mercure (Montréal); Ensemble de la SMCQ, Walter Boudreau, direction.

\section{7}

- *Serge Arcuri, Fragments (1997), piano. Création : 12 juin, Orient-Express (concert 239), Salle Pierre-Mercure (Montréal); Louise-Andrée Baril, piano.

\section{8}

- "John Oswald, Homonymy (1998), flûte, hautbois, clarinette, basson, cor, trombone, percussion, violon, violoncelle, contrebasse et bande vidéo. Création: $1^{\mathrm{er}}$ mai, Bédéphonie, Salle Bandeen (Sherbrooke); Ensemble de la SMCQ, Walter Boudreau, direction.

- "Chris Paul Harman, Procession burlesque (1998), piano, percussions, 2 violons, alto, violoncelle et contrebasse. Création : 13 novembre, Long-courrier (concert 246), Salle Pollack (Montréal); Ensemble de la SMCQ, Walter Boudreau, direction.

\section{9}

- "Jean Lesage, Le livre des mélancolies (1999), quatuor à cordes et clarinette. Création: 11 novembre, Concert invitation (concert 252), Salle Pierre-Mercure (Montréal); Ensemble Musica Nova, Pierre Simard, direction.

- Créations du 9 décembre, Triplé (concert 253), Salle Pierre-Mercure (Montréal):

- Vincent Collard, Concerto «Lucky Luke» (1999), trombone ténor et grand ensemble. Alain Trudel, trombone, Ensemble de la SMCQ, Walter Boudreau, direction.

- " Isabelle Panneton, Volando (1999), violon et grand ensemble. Julie-Anne Derome, violon, Ensemble de la SMCQ, Walter Boudreau, direction.

\section{0}

- Serge Arcuri, Walter Boudreau, Vincent Collard, Yves Daoust, Alain Dauphinais, André Duchesne, Louis Dufort, Sean Ferguson, Michel Gonneville, André Hamel, Alain Lalonde, Estelle Lemire, Jean Lesage, Luc Marcel, Marie Pelletier, John Rea, Anthony Rozankovic et Gilles Tremblay, Symphonie du millénaire (2000), 333 musiciens, 2000 carillonneurs, 15 clochers, grand orgue, carillon de 56 cloches et 2 camions de pompiers. Création : 3 juin, Oratoire Saint-Joseph (Montréal) ${ }^{8}$.

8. Détail des effectifs artistiques: www.smcq.qc.ca/smcq/fr/symphonie/ effectifs/ (consulté le 15 juin 2017).

- Ana Sokolović, Bouba ou Les tableaux d'une exposition (2000), conte musical pour 2 acteurs et 9 instruments. Livret: Marie Décary. Commandée avec l'aide du CALQ. Création: 7 décembre, Bouba ou Les tableaux d'une expédition, Salle Pierre-Mercure (Montréal) ; Catherine Trudeau et Sébastien Rajotte, comédiens, Ensemble SMcQ Jeunesse, Pierre Simard, direction.

\section{1}

- *Vincent Collard, Destin anémique (Voyage au centre du vide) (2000), cor et ensemble. Création: 8 mars, Sur terre comme au ciel (concert 257), Salle PierreMercure (Montréal); Michèle Gagnon, cor, Ensemble de la SMcQ, Walter Boudreau, direction. 
- Créations du 6 mars, MusiMars 2002 : les ailes du désir (concert 26o), Salle Pollack (Montréal) :

- "Denis Gougeon, Clere Vénus (2001), soprano, flûtes (en do et en sol), hautbois, clarinette en si bémol, clarinette basse, basson, 2 cors, piano et cordes. Texte : sonnets de Louise Labé. Marie-Danielle Parent, soprano, Ensemble de la SMCQ, Walter Boudreau, direction.

- *Melissa Hui, Come as You Are (2000), pipa et orchestre de chambre. Liu Fang, pipa, Ensemble de la SMCQ, Walter Boudreau, direction.

- "John Rea, J'ignore si j'étais un homme rêvant alors que j'étais un papillon ou si je suis à présent un papillon rêvant que je suis un homme ou Homme papillon (2001), Yamaha Disklavier et orchestre de chambre. Ensemble de la SMCQ, Walter Boudreau, direction.

- Créations du 17 octobre, Trajectoires (concert 262), Salle Pierre-Mercure (Montréal):

- *Justin Mariner, Apparent Motions (2002), ensemble à cordes, célesta, 2 pianos et percussions. Ensemble de la SMCQ, Walter Boudreau, direction.

- "Smith, Linda Catlin, Path of Uneven Stones (2000), ensemble et piano principal. Ensemble de la SMCQ, Walter Boudreau, direction.

\section{3}

- *Paul Steenhuisen, Les enfers éternels des gens désespérés (2002), 4 voix. Livret: Christian Bök. Commandée pour le Hilliard Ensemble9. Création : 4 mars, Les voix à l'honneur, Salle Pollack (Montréal).

- Michel Viau et Johanne Latreille, TapaJungle! (2003), 1 récitant et 4 percussions. Création : 10 octobre, TapaJungle!, Maison de la culture Mercier (Montréal); Félix Beaulieu-Duchesneau, comédien, QUAD percussions originales ${ }^{10}$.

- "Michael Oesterle, Anytime (2003), 2 saxophones sopranos, 2 saxophones altos et 4 percussions. Création: 17 novembre, Sax, tambours et cie (concert 266), Salle Pierre-Mercure (Montréal); quatuor de saxophones Quasar ${ }^{11}$, QUAD percussions originales, Walter Boudreau, direction.

\section{4}

- *Howard Bashaw, Double entente (2002), trombone, tuba, piano et ensemble. Création: 4 mars, MusiMars 2004: modulations de fréquence, Salle Pollack (Montréal); Louise-Andrée Baril, piano, Yan Sallafranque, tuba, Alain Trudel, trombone, Ensemble de la SMCQ, Walter Boudreau, direction.

\section{5}

- *Yves Daoust, Le musicolateur (2005), animateur-compositeur et participants, machine à composer. Création : 28 février, Le musicolateur, Salle Pierre-Mercure (Montréal); Félix Boisvert et Yves Daoust, animateurs-compositeurs.

- *Serge Provost, Le Stelle (Les étoiles) (2004), quatuor à cordes et quatuor vocal. Commandée pour le Hilliard Ensemble et le Quatuor Bozzini ${ }^{12}$. Création : 2 mars, Montréal/Nouvelles Musiques 2005: Hilliard Ensemble, Quatuor Bozzini, Salle Pierre-Mercure (Montréal).
9. Voir: www.smcq.qc.ca/smcq/fr/ artistes/h/hilliardensemble_(consulté le 6 avril 2017).

10. Michel Viau, Stéphanie Dionne, Jean-François Côté, Johanne Latreille et Charles Duquette.

11. Voir: www.quasar4.com (consulté le 21 mars 2017). Le 25 novembre 2004 , l'œuvre est-recréée sous une nouvelle mouture par Quasar et porte le titre sometime.

12. Voir: www.quatuorbozzini.ca (consulté le 6 avril 2017). 
13. L'œuvre fait l'objet d'une révision en 2012.

14. Voir: https://theatrequivala. wordpress.com (consulté le 15 juin 2017).

15. Ébauche préliminaire de Toy (Music Box), créée le 4 mai 2010 par l'Orchestre philharmonique de RadioFrance à l'Opéra de Shanghai, sous la direction de François-Xavier Roth - les solistes sont les mêmes qu'à la création 64

\section{6}

- Créations du 3 mars, MusiMars 2006: aurores boréales, Salle Pollack (Montréal): - Marc Hyland, Rosarium (2005), baryton, piano, flûte et harpe. Commandée avec l'aide du Fonds Gilles Tremblay. Rolf Bertsch, piano, Lise Daoust, flute, Vincent Ranallo, baryton, Anabelle Renzo, harpe, Walter Boudreau, direc$\operatorname{tion}^{13}$.

- *Yannick Plamondon, L'ange casseur [version concert] (2002-2005), soprano et orchestre de chambre. Julieanne Klein, soprano, Ensemble de la SMCQ, Walter Boudreau, direction.

\section{7}

- *Benoit Côté, La fugue (2007), 4 acteurs-chateurs-récitants, bande et instruments inventés. Création : 15 janvier, La fugue, Maison de la culture HochelagaMaisonneuve (Montréal); Théâtre Qui Va Là ${ }^{14}$.

- Michel Gonneville, HoMa (2007), 24 instruments à vent, 5 percussions et grand orgue. Création : 27 septembre, 9 e Festival d'automne, Série hommage Claude Vivier: musiques du feu (concert 285), Église Saint-Jean-Baptiste (Montréal); JeanWilly Kunz, orgue, Ensemble de la SMCQ, Walter Boudreau, direction.

\section{8}

- Alain Lalonde, Expirespoirespire? (1995-2008), trombone solo et 25 interprètes en groupes instrumentaux interagissants (flûte, hautbois, clarinette, basson, cor, trompette, trombone, 2 percussions, 8 violons, 2 alti, 3 violoncelles et 2 contrebasses). Création: 7 mars, Les arches de lumière (concert 288), Salle Pollack (Montréal); Alain Trudel, trombone, Ensemble de la SMCQ, Walter Boudreau, direction.

- Créations du 24 avril, La (re)création du monde (concert 290), Salle Pierre-Mercure (Montréal) :

- "Nicolas Gilbert, Circuits parallèles (2007), orchestre de chambre. Ensemble de la SMCQ, Walter Boudreau, direction.

- "Estelle Lemire, Mémoire / Éclaircie (2008), grand ensemble. Ensemble de la SMCQ, Walter Boudreau, direction.

\section{9}

- Créations du 11 mai, Shanghai Spring International Music Festival 2009, Opéra de Shanghai; toutes les œuvres ont été commandées avec le soutien du CALQ, du CAC et de la Fondation SOCAN :

- "Simon Bertrand, Duels (2009), double concerto pour premier violon, erhu et orchestre. Ma Xianghua, erhu, Orchestre symphonique de Shanghai, Zhang $\mathrm{Yi}$, direction.

- "José Evangelista, O Qin (2009), guqin, yangqin et orchestre. Wang Long, guqin et yangqin, Orchestre symphonique de Shanghai, Zhang Yi, direction.

- "Denis Gougeon, Toy (Prceludium Mechanicus) ${ }^{15}$ (2008-2009), 2 dizis (flûtes de bambou) et grand orchestre. Commande: Festival Présences-SmcQ. Qian Jun et Jin Kai, dizis, Orchestre symphonique de Shanghai, Zhang Yi, direction. 
- *Analía Llugdar, In the Rays of the Oriental Pearl (2009), 2 erhu, 2 zhonghu et orchestre. Zhao Lei et Aiai, erhu, Luo Yuanyuan et Chan Xiaoyan, zhonghu, Orchestre symphonique de Shanghai, Zhang Yi, direction.

- "Pierre Michaud, Carnet de voyage (2009), dizi (bangdi et qudi), pipa, orchestre et traitement. Qian Jun, dizi, Tang Xiaofeng, pipa, Orchestre symphonique de Shanghai, Zhang Yi, direction.

- *Farangis Nurulla-Khoja, Recitative of Nightingale (2009), zhonghu, sheng, suona et orchestre. Chen Xiaoyan, zhonghu, Zhao Zhen, sheng, Zhang Quinyuan, suona, Orchestre symphonique de Shanghai, Zhang Yi, direction.

- *Sean Pepperall, Happy Green Tea-Quake's Toll-Resilience (2009), erhu, guzheng et orchestre. Duan Aiai, erhu, Liu Le, guzheng, Orchestre symphonique de Shanghai, Zhang Yi, direction.

- "Serge Provost, From the Lake to the Sky (2009), guanzi, guzheng et orchestre. Zuo Yiwei, guanzi, Liu Le, guzheng, Orchestre symphonique de Shanghai, Zhang Yi, direction.

\section{0}

- "David Adamcyk, Devant l'azur (2008), soprano, saxophone ténor, saxophones baryton et piano. Création : 18 mars, Série hommage Gilles Tremblay: Solstices, Salle Pierre-Mercure (Montréal); Zosha Di Castri, piano, Adam Kinner, saxophones, Julieanne Klein, soprano.

- "Wolf Edwards, Irons (2009-2010), saxophones, contrebasson, percussions et cordes. Création : 28 octobre, Halloween, Salle Pierre-Mercure (Montréal); Ensemble de la SMCQ, ensemble de percussions Sixtrum ${ }^{16}$, quatuor de saxophones Quasar, Walter Boudreau, direction.

\section{1}

- Walter Boudreau et Yves Daoust, Le téléphone bien tempéré (2011), clavecin, orgue, quintette à vent, sonneries de cellulaires et support. Création : 20 février, Apportez votre cellulaire, Église Saint-Jean-Baptiste (Montréal); Marc Béland, animateur, Geneviève Soly, clavecin, Jean-Willy Kunz, orgue, Ensemble de la SMCQ, Walter Boudreau, direction, Simon Bertrand, João Catalão, Denis Chabot, Albert Devito, Denis Dion, Nicolas Gilbert, Joane Hétu, Diane Labrosse, Alain Lalonde, Sean Pepperall, Robert Slapcoff et Ana Sokolović, assistants du chef, avec public participant et cellulaires.

\section{2}

- Nicolas Gilbert, L'état des lieux (2011-2012), soprano, clarinette, trombone, percussions, alto et traitement. Commandée pour l'ensemble instrumental Ars Nova ${ }^{17}$. Création: 12 avril, Concert, Hippodrome Scène nationale (Douai, France); Géraldine Keller, soprano.

- Nicole Lizée, The Man With the Golden Arms (2012), batterie, orchestre de chambre et 6 percussions. Création: 13 septembre, L'homme et son désir, Salle Pierre-Mercure (Montréal); Ben Reimer, batterie, ensemble de percussions Sixtrum, Ensemble de la SMCQ, Walter Boudreau, direction.
16. Voir: www.sixtrum.com (consulté le 21 mars 2017)

17. Voir: www.arsnova-ensemble.com (consulté le 21 mars 2017). 
- *Alex Pauk, Musiques immergées (2011), 27 musiciens et support. Création: 10 novembre, Chutes libres, Salle Pierre-Mercure (Montréal); Ensemble de la SMCQ, Walter Boudreau, direction.

\section{3}

- Félix Boisvert et Benoit Côté, Concerto au sol (2013), mime, bande et électroacoustique. Création : 8 octobre, Concerto au sol, Théâtre Aux Écuries (Montréal); Félix Boisvert, interprétation.

\section{5}

- *Benoit Côté, Fonoformies (2015) saxophone, voix, violon, violoncelle et parcours. Création: $1^{\text {er }}$ mars, Fonoformies (Montréal/Nouvelles Musiques 2015), Chapelle historique du Bon-Pasteur (Montréal); Emmanuelle Lizère, voix, violon et violoncelle, Louis-Philippe Bonin, saxophone.

- Michel Seta, Touch E Flurry (2015) tu-yo télescopique, membranes vibrantes, The Pipe et flûtes over-tone. Création: $1^{\text {er }}$ mars, L'air du temps (Montréal/Nouvelles Musiques 2015), Université Concordia, Hexagram Black Box (Montréal).

- Simon Bertrand, La chasse-galerie (2015), quatuor de saxophone, orchestre et récitant. Création: 11 juin, Le concert du printemps de l'OSDL, cocathédrale SaintAntoine-de-Padoue (Longueuil); Jocelyn Lebeau, récitant, quatuor de saxophones Quasar, Orchestre symphonique de Longueuil, Marc David, direction.

- Silvio Palmieri, Ossessione (Poema Concertante) (2015), piano et ensemble. Commandée pour la pianiste-égérie Louise Bessette et pour l'Ensemble de la SMCQ, avec l'aide du CALQ. Création: 25 septembre, Série hommage John Rea: L'homme papillon, Salle Pierre-Mercure (Montréal); Walter Boudreau, direction.

\section{7}

- "Sandeep Bhagwati, Niemandslandhymnen (2017), œuvre multidisciplinaire. Commandée avec l'aide du CALQ. Création : 18 mai, Niemandslandhymnen, Usine C (Montréal); Suzie LeBlanc, soprano, Marie-Annick Béliveau, mezzosoprano, Sameer Dublay, chanteur khyal, Gabriel Dharmoo, chant expérimental, Nicholas Isherwood, baryton-basse, Guy Pelletier, flûtes, Wu Wei, sheng, Lori Freedman, clarinette basse, Felix Del Tredici, trombone basse, Aiyun Huang, percussions, Joseph Browne, électroacoustique, et Angélique Willkie, danse, actrice. 